\title{
Establishment of Dynamic Model of axle Box Bearing of high-speed Train under Variable Speed Conditions
}

Yongqiang Liu ( $\nabla$ liuyq@stdu.edu.cn )

Shijiazhuang Tiedao University

Baosen Wang

Shijiazhuang Tiedao University

Bin Zhang

University of South Carolina College of Engineering and Computing

Shaopu Yang

Shijiazhuang Tiedao University

\section{Research Article}

Keywords: Variable speed conditions, High-speed train, Bearing model, Angle iteration, Order ratio spectrum

Posted Date: July 7th, 2021

DOl: https://doi.org/10.21203/rs.3.rs-656308/v1

License: (c) (i) This work is licensed under a Creative Commons Attribution 4.0 International License. Read Full License 


\title{
Establishment of dynamic model of axle box bearing of high-speed train under variable speed conditions
}

\author{
Yongqiang Liu ${ }^{1, *}$, Baosen Wang ${ }^{2,3}$, Bin Zhang ${ }^{3}$, Shaopu Yang ${ }^{4}$ \\ 1. School of Mechanical Engineering, Shijiazhuang Tiedao University, Shijiazhuang, \\ 050043, China. \\ 2. School of Traffic and Transportation, Shijiazhuang Tiedao University, Shijiazhuang, \\ 050043, China. \\ 3. College of engineering and computing, University of South Carolina, Columbia, South \\ Carolina, 29208, America. \\ 4. State Key Laboratory of Mechanical Behavior and System Safety of Traffic \\ Engineering Structures, Shijiazhuang Tiedao University, Shijiazhuang 050043, China. \\ *corresponding author: Yongqiang Liu; liuyq@stdu.edu.cn
}

\begin{abstract}
This paper establishes a dynamic model of the bearing rotor system of a high-speed train under variable speed conditions. Different from previous works, the proposed model simplifies the contact stress and considers the compensation balance excitation caused by the rotor mass eccentricity. The angle iteration method is used to solve the challenging problem that the roller space position cannot be determined in bearing rotation. The simulation results show that the model accurately describes the dynamics of bearing under varying speed profiles that contain acceleration, deceleration and speed oscillation stages. The order ratio spectrum of the bearing vibration signal indicates that both single frequency and multiple frequency in simulation results are consistent with that in theoretical results. Experiments of bearing with outer ring fault and inner ring fault under various operating conditions are presented to verify the developed model.
\end{abstract}

Keywords: Variable speed conditions, High-speed train, Bearing model, Angle iteration, Order ratio spectrum

\section{Introduction}

In recent years, with the rapid development of high-speed railways, the 3 global high-speed railway mileage and the number of trains increased rapidly, 
which make safe and reliable operation of train critical to railway safety [1,2]. As key components of train running systems, bogie axle box bearings have attracted extensively studies on movement modeling and state monitoring [3]. To better understand the mechanism of bearing faults and their effects on movement, dynamic simulation of bearing systems are commonly used. The bearing model studies the influence of parameters on bearing motion states by establishing a set of differential equations, which can be solved by using numerical solution methods.

In the early stage of research, relatively simple nonlinear equations were used to simulate bearing motion, and study the motion law of bearings based on the exact solutions of these equations. For instance, Chinta [4] used nondimensional differential equations to describe the unbalanced response of rotor bearings, and used Floquet theory to study the stability and bifurcation behavior of bearing systems. Sinou [5] established a dynamic model of a flexible rotor-rolling bearing system under unbalanced excitation, used the harmonic balance method to study the system's nonlinear motion law, and analyzed the influence of bearing radial clearance and unbalanced mass on Hertzian contact force between roller and raceway.

Since traditional nonlinear equation solving methods can only obtain approximate solutions of nonlinear system with fewer degree of freedom (DOF), some more advanced methods were developed for higher DOF bearing systems. Fukata and Gad et al [6] used a numerical method to solve the differential equations of ball bearing motion for the first time, and intuitively analyzed the motion state of bearings. Tiwari and Gupta et al [7] established a nonlinear dynamic model of bearing rotor systems, and analyzed the influence of radial clearance on the motion stability based on the Floquet theory. Sawalhi et al [8] built a dynamic model of bearing and gear coupling system, in which a small sprung mass with relatively high damping is used to represent a typical high frequency. Furthermore, the model is verified through experiments under different fault conditions [9]. Tsuha et al [10] developed a rolling bearing model with improved fidelity by introducing a set of equivalent contact stiffness and damping and using the theory of elastic fluid lubrication to calculate the contact force. Song [11] put forward a dynamic model of spindle bearing systems by combining angular contact bearing and floating bearing. Compared with transitional methods, these advanced methods consider more factors that affect bearing motion, so the established bearing motion model is more accurate. At the same time, the numerical solution method makes the solution of the equation more intuitive 
and provides great convenience for further nonlinear analysis. However, these works focus on bearing under constant operating conditions, which cannot sufficiently describe the dynamics operating conditions.

Different from those bearings working in relatively consistent operating conditions, high-speed train bearings in the running system often work in high-speed and heavy-duty harsh conditions and are severely affected by strong noises in the surrounding environment. It is difficult to directly evaluate the quality of the bearings and study the bearing motion states. To address these challenges, the dynamic model simulation methods are desired for bearings of high-speed trains. The method of dynamic modeling should consider the influence of various factors on the motion states of bearings, greatly reducing the research cost, and improving the research efficiency. Cao et al [12] established a nonlinear dynamic model of the CRH1 EMU (China Railway High-speed-1, Electric Multiple Units) coupling system, which considers the loose fit between the inner ring and the main shaft of the axle box bearing of the high-speed train. They also studied and compared the motion characteristics of the model by using optimized numerical algorithms under various working conditions. Cao and He et al [13] established a dynamic model of high-speed rolling bearings by considering actual factors such as centrifugal expansion and thermal expansion of the inner ring. The model was then used to predict some system parameters such as contact angle and bearing stiffness and conduct correlative analysis of bearing damage mechanism. Yang et al [14] established a 4 DOF nonlinear dynamic model with classic fault of high-speed train bearing-rotor systems. The model was verified by a certain type of high-speed train bearing under outer ring, inner ring and roller element fault conditions. Liu et al [15] established a 12 DOF non-linear dynamic model of high-speed train axle box bearings with outer ring faults, in which the influence of bearing speed and fault size on system motion was studied from a nonlinear perspective. Wang et al [16] proposed a novel stochastic vehicle-track coupled model to evaluate the dynamic performance of axle box bearings in a high-speed train with unsteady wind load and random track irregularities and study the influence of crosswind speed on dynamic performance of bearings.

With these successes in bearing model research, the limitation of existing works, however, is that failure mechanism and bearing motion are all studied with static bearing speed. Mishra et al [17] established a bond graph model of rolling element bearings in special operating conditions, and verified the effectiveness through experiments [18]. The main function of this model 
is to generate a series of non-stationary signals for fault diagnosis algorithm research. However, this model cannot describe the vibration response of each part of bearing rotor systems. Moreover, these bearing models have a single structure, which is insufficient to represent the structural characteristics of tapered roller bearings of high-speed trains. Furthermore, in addition to the bearing mass eccentricity, the shaft mass eccentricity is also very important but is always ignored in bearing rotor systems. To address these limitations, this paper establishes a dynamic model of coupling systems of bearing and shaft to accurately simulate the dynamics of real axle box bearings of highspeed trains under variable speed conditions. The novelty of the research is three-fold: First, the shaft and two bearings are regarded as a coupled system in which the shaft is regarded as the rotor in this system and the mass eccentricity of the shaft is considered; Second, the angular iteration method is used to calculate the spatial position of the bearing rollers at each moment under the variable speed operation of the bearing; Third, the model is verified through a series of simulation and actual experiments with different fault modes.

The paper is organized as follows: Section 2 describes the process of building a shaft-bearing coupling system model under conditions of variable speed and introduces the outer ring fault, inner ring fault and rolling element fault models. Section 3 shows a few simulation results when the model has different type of fault under variable speed condition. Section 4 verifies the effectiveness of the model by comparing actual experiments and simulation experiments.

\section{Bearing Model}

\subsection{Bearing dynamic model}

The running part of a high-speed train consists of an axle, a left wheel, and a right wheel. According to the law of conservation of energy, the axle mass is equivalent to the axle center of mass. Fig. 1 shows the structure of the axle box bearing of high-speed trains, in which $O_{1}, O_{2}, O_{3}$ are the bearing geometry center, center of shaft section and center of mass of shaft section, respectively; $e$ is the eccentricity of cross section of the shaft, $m_{c}$ is the equivalent mass $(\mathrm{kg})$ of the shaft at the center; $K, C$ are the stiffness and damping of the shaft, respectively; $F_{x \mathrm{~L}}, F_{y \mathrm{~L}}$ are the reaction force $(\mathrm{N})$ received by the left end bearing in the horizontal and vertical directions, respectively, $F_{x \mathrm{R}}, F_{y \mathrm{R}}$ are the reaction force $(\mathrm{N})$ received by the right end 
bearing in the horizontal and vertical directions, respectively, and $\alpha$ and $\beta$ are the contact angle and the half cone angle given in degree, respectively. Since the rollers of tapered roller bearings change the direction of contact force and decompose the load on the bearing into axial and radial directions, the half cone angle should be considered in the model.

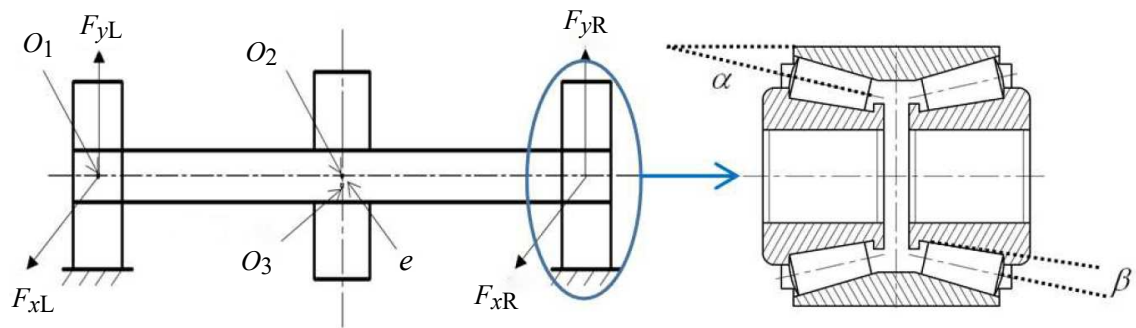

Fig. 1: Shaft and bearing coupling system model

Fig. 2 shows the schematic diagram of bearings dynamics model, in which $m_{1}, m_{2}, m_{b}$ are the masses $(\mathrm{kg})$ of the bearing inner ring, bearing and unit resonator, respectively; $x_{1}, y_{1}$ are displacement $(\mathrm{m})$ of the left row inner ring in the horizontal and vertical directions, respectively; $K_{1}, C_{1}$ are support stiffness $(\mathrm{N} / \mathrm{m})$ and support damping $(\mathrm{N} \cdot \mathrm{s} / \mathrm{m})$ of the inner ring, respectively; $x_{2}, y_{2}$ are the displacement $(\mathrm{m})$ of the outer ring in the horizontal and vertical directions, respectively; $K_{2}, C_{2}$ are the support stiffness $(\mathrm{N} / \mathrm{m})$ and support damping $(\mathrm{N} \cdot \mathrm{s} / \mathrm{m})$ of the outer ring, respectively; $y_{b}, K_{b}, C_{b}$ are the vertical displacement $(\mathrm{m})$, stiffness $(\mathrm{N} / \mathrm{m})$ and damping $(\mathrm{N} \cdot \mathrm{s} / \mathrm{m})$ of the unit resonator, respectively. The bearing in Fig. 2 is considered as a model that is coupled with a series of spring mass models and is simulated by establishing a set of differential equations according to Newton's second law.

When a fault occurs, the periodic impact of the fault leads to natural vibration of bearing inner and outer rings and other components. The vibrations can be accurately simulated by adjusting the stiffness and damping coefficient of unit resonator $[19,20]$. According to Newton's second law, the dynamics of a bearing-rotor system can be described as:

$$
\left\{\begin{array}{l}
m_{c} \ddot{x}_{c}+C \dot{x}_{c}+K\left(x_{c}-x_{\mathrm{r} 1}\right)+K\left(x_{c}-x_{11}\right)=m_{c} e w_{c}^{2} \cos \left(w_{c} t\right) \\
m_{c} \ddot{y}_{c}+C \dot{y}_{c}+K\left(y_{c}-y_{\mathrm{r} 1}\right)+K\left(y_{c}-y_{11}\right)=m_{c} e w_{c}^{2} \cos \left(w_{c} t\right)-m_{c} g
\end{array}\right.
$$




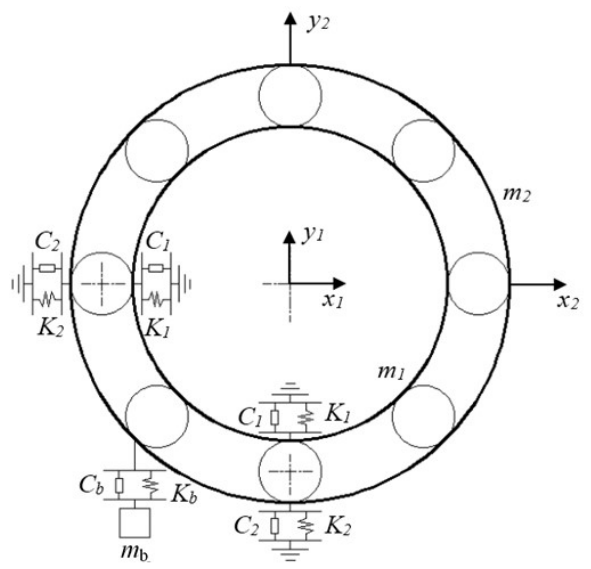

Fig. 2: Spring mass system of simplified bearings

$$
\begin{aligned}
& \left\{\begin{array}{l}
m_{1} \ddot{x}_{\mathrm{r} 1}+C_{1} \dot{x}_{\mathrm{r} 1}+K_{1} x_{\mathrm{r} 1}+F_{\mathrm{xR}} \cos (\alpha-\beta)=0 \\
m_{1} \ddot{y}_{\mathrm{r} 1}+C_{1} \dot{y}_{\mathrm{r} 1}+K_{1} y_{\mathrm{r} 1}+F_{\mathrm{yR}} \cos (\alpha-\beta)=0 \\
m_{2} \ddot{x}_{\mathrm{r} 2}+C_{2} \dot{x}_{\mathrm{r} 2}+K_{2} x_{\mathrm{r} 2}+F_{\mathrm{xR}} \cos (\alpha+\beta)=0 \\
m_{2} \ddot{y}_{\mathrm{r} 2}+\left(C_{2}+C_{\mathrm{b}}\right) \dot{y}_{\mathrm{r} 2}+\left(K_{2}+K_{\mathrm{b}}\right) y_{\mathrm{r} 2}-K_{\mathrm{b}} y_{\mathrm{b}} \\
\quad-C_{\mathrm{b}} \dot{y}_{\mathrm{b}}-F_{\mathrm{yR}} \cos (\alpha+\beta)=F \cos (\alpha+\beta)
\end{array}\right. \\
& m_{\mathrm{b}} \ddot{y}_{\mathrm{rb}}+C_{\mathrm{b}}\left(\dot{y}_{\mathrm{rb}}-\dot{y}_{\mathrm{r} 2}\right)+K_{\mathrm{b}}\left(y_{\mathrm{rb}}-y_{\mathrm{r} 2}\right)=0 \\
& \left\{\begin{array}{l}
m_{1} \ddot{x}_{11}+C_{1} \dot{x}_{11}+K_{1} x_{11}+F_{\mathrm{xL}} \cos (\alpha-\beta)=0 \\
m_{1} \ddot{y}_{11}+C_{1} \dot{y}_{11}+K_{1} y_{11}+F_{\mathrm{yL}} \cos (\alpha-\beta)=0 \\
m_{2} \ddot{x}_{12}+C_{2} \dot{x}_{12}+K_{2} x_{12}+F_{\mathrm{xL}} \cos (\alpha+\beta)=0 \\
m_{2} \ddot{y}_{12}+\left(C_{2}+C_{\mathrm{b}}\right) \dot{y}_{12}+\left(K_{2}+K_{\mathrm{b}}\right) y_{12}-K_{\mathrm{b}} y_{\mathrm{b}} \\
\quad-C_{\mathrm{b}} \dot{y}_{\mathrm{b}}-F_{\mathrm{yL}} \cos (\alpha+\beta)=F \cos (\alpha+\beta)
\end{array}\right. \\
& m_{\mathrm{b}} \ddot{y}_{1 \mathrm{~b}}+C_{\mathrm{b}}\left(\dot{y}_{1 \mathrm{~b}}-\dot{y}_{12}\right)+K_{\mathrm{b}}\left(y_{1 \mathrm{~b}}-y_{12}\right)=0
\end{aligned}
$$

where $x_{c}, y_{c}$ are the displacement of centroid of the shaft in the horizontal and vertical directions, respectively; $x_{\mathrm{r} 1}, y_{\mathrm{r} 2}$ are the displacement of the outer ring and the inner ring at the right end bearing in the horizontal direction, respectively; $y_{\mathrm{r} 1}, y_{\mathrm{r} 2}$ are the displacement of the outer ring and the inner ring at the right end bearing in the vertical direction, respectively; $x_{11}, x_{12}, y_{11}, y_{12}$ are for the left end bearing; $y_{\mathrm{rb}}, y_{\mathrm{lb}}$ are the displacement of the unit resonator 
at right and left end in the vertical direction, respectively; $F$ is axle load of train. In this model, Eq. (1) is the vibration equations of the shaft in the horizontal and vertical directions; Eq. (2) is the vibration equations of the right end bearing in the horizontal and vertical directions; Eq. (4) is the vibration equations of the left end bearing in the horizontal and vertical directions; Eqs. (3) and (5) are the vibration equations of the unit resonator in the vertical direction.

\subsection{Bearing support reaction force}

In the model, bearing support reaction force is needed. However, the magnitude and direction of the force change with the rotation of the bearing. The force can be calculated as follows:

1. Calculating the contact force on each roller.

2. Decomposing the force into the forward direction and radial direction of the bearing.

3. Combining all the force in the above two directions and the bearing support reaction force can be obtained in these two directions.

Since the forces on the inner and outer rings of bearings are transmitted through the rollers in bearing movement, the reaction force is the sum of the resultant force of the contact forces at each roller. In practice, the motion state and force of bearings are very complicated. To simplify the calculation, the following assumptions are introduced. Note that these assumptions are commonly used and do not lower the accuracy and fidelity of the model.

1. The outer ring of bearings is fixed on the rigid element with rotating velocity of 0 and it has lateral and vertical displacements.

2. The rolling elements are equidistantly located on the raceway and perform pure rolling (i.e. sliding of rolling elements are not considered);

3. The contact stress is considered in the form of Hooke's law.

Fig. 3 shows the side view of a double-row tapered roller bearing with $N_{0}$ rollers that are equally separated by an angle of $2 \pi / N_{0}$. In the rotating operation, the angle of the $i$-th roller $\left(i=1,2, \ldots, N_{0}\right)$ turns at time $t$ can be calculated as: 


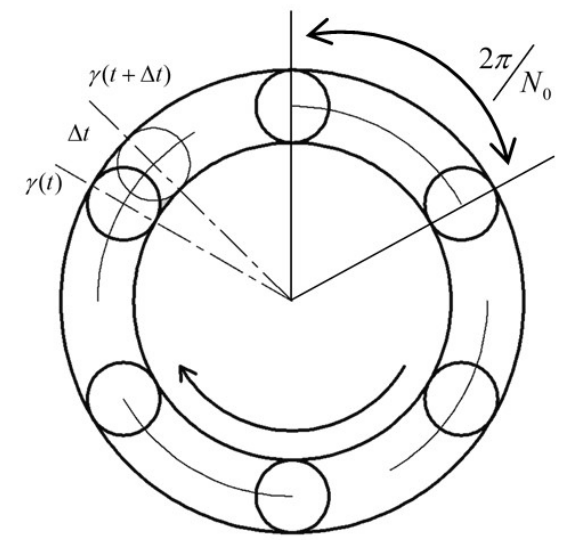

Fig. 3: Side view of a double-row tapered roller bearing

$$
\theta_{i}=\gamma(t)+\frac{2 \pi}{N_{0}}(i-1), i=1,2, \ldots, N_{0}
$$

where $\gamma(t)$ is the angle that the roller indexed by 1 turns in time $t$, and its initial location is $\gamma\left(t_{0}\right)=0$. The normal contact deformation of the $i$-th roller of the right end bearing with the raceway at the angular displacement $\theta_{i}$ can be calculated as:

$$
\delta_{i}=\left(x_{\mathrm{r} 1}-x_{\mathrm{r} 2}\right) \cos \theta_{i}+\left(y_{\mathrm{r} 1}-y_{\mathrm{r} 2}\right) \sin \theta_{i}-c_{0}
$$

where $c_{0}$ is the bearing radial clearance. The contact force between the ball and the raceway can be expressed by the nonlinear Hertz's contact force as $[21,22]$ :

$$
P_{i}=K_{\mathrm{t}} \delta_{i}^{n} H_{i}
$$

where $K_{\mathrm{t}}$ is the contact stiffness, $n=10 / 9$. When $\delta_{i}>0, H_{i}=1$ and it indicates that there is a nonlinear Hertz's contact force while $\delta_{i} \leq 0, H_{i}=0$ indicates that there is no nonlinear Hertz's contact force. By decomposing the contact force at every roller into the horizontal and vertical directions, the resultant force in the two directions can be obtained. The total contact force of the bearing in the horizontal and vertical directions is the sum of the resultant force from all rollers, which is given as: 


$$
\left\{\begin{array}{l}
F_{\mathrm{xR}}=K_{\mathrm{t}} \sum_{i=1}^{N_{0}} \delta_{i}^{\frac{10}{9}} H_{i} \cos \theta_{i} \\
F_{\mathrm{yR}}=K_{\mathrm{t}} \sum_{i=1}^{N_{0}} \delta_{i}^{\frac{10}{9}} H_{i} \sin \theta_{i}
\end{array}\right.
$$

where $F_{\mathrm{xR}}, F_{\mathrm{yR}}$ are the contact force in horizontal and vertical directions, respectively. Similarly, $F_{\mathrm{xL}}, F_{\mathrm{yL}}$ can be obtained.

\subsection{Model analysis with different fault modes}

To solve the model, the angle $\gamma(t)$ that the roller rotates at any time instant $t$ is needed. However, for high-speed train, the angular rotating velocity of the main shaft $w_{0}(t)$ is not a constant. To address this, we simplify and apply the angle iteration method [17] to calculate the angle $\gamma(t)$ in real time.

The angle $\gamma(t)$ can be calculated as:

$$
\gamma(t)=\int_{0}^{+\infty} w_{0}(t) t \mathrm{dt}
$$

When the rotation speed changes with time, $\gamma(t)$ cannot be expressed as a linear function of time and the total angle that the roller turns at each moment cannot be determined by the angular velocity.

The proposed angle iteration method assumes that the bearing speed is piecewise constant, i.e., the speed is constant within a short period time $\Delta t(\Delta t \rightarrow 0)$. As a result, the angle that the bearing rotates at time $t+\Delta t$ can be expressed as $\gamma(t+\Delta t)=\gamma(t)+w(t) \Delta t$, where $w(t)$ is the relative speed of the roller with respect to the fault location, which can be on the outer ring, inner ring, and roller itself. Therefore, $w(t)$ has different values for different types of faults. When the fault is on the outer (or inner) ring, $w(t)$ is the relative speed of the roller to the outer (or inner) ring at $t$. When the fault is on the rolling element, $w(t)$ is the rotating speed of the roller itself at $t$. The schematic diagram is shown in Fig. 3. Then the problem transforms to the estimation of $w(t)$. In the simulation, $\Delta t$ represents the step size used in each iteration of the ode 45 function.

\subsubsection{Model of outer ring fault}

Fig. 4 shows the case when the outer ring has a fault $[23,24]$ located in the lower bearing area, in which $\theta_{\mathrm{c}}$ is the location of the fault center and $\theta_{\mathrm{ca}}$ 


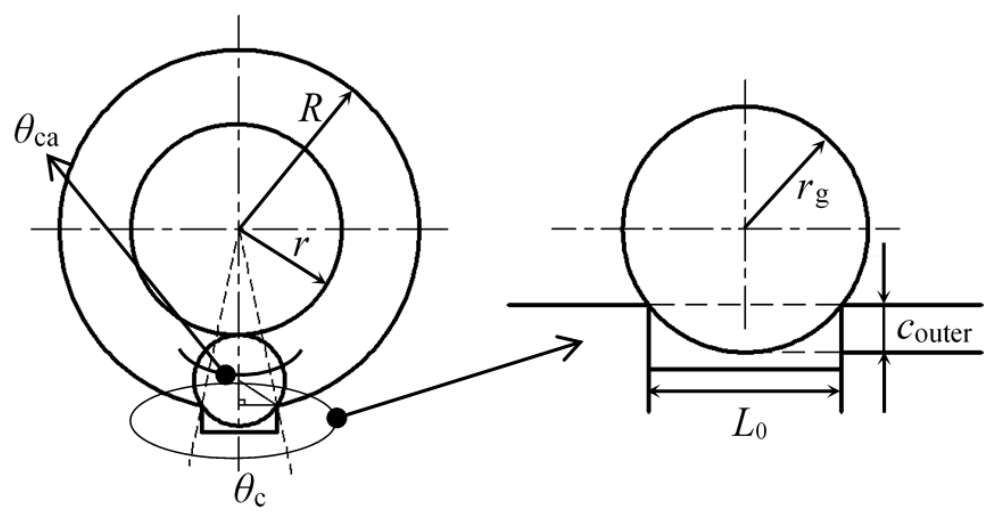

Fig. 4: Model of outer ring fault

is the central angle corresponding to the arc where the fault is located, $L_{0}$ is the fault width, $r$ is the inner ring radius of bearing, $R$ is the outer ring radius of bearing, $r_{\mathrm{g}}$ is the section radius at the center of mass of tapered roller. When a roller rolls over the fault area, the roller descending depth $c_{\text {outer }}$ between the roller and the raceway can be expressed by:

$$
c_{\text {outer }}= \begin{cases}r_{\mathrm{g}}-\sqrt{r_{\mathrm{g}}^{2}-\left(\frac{L_{0}}{2}\right)^{2}}, & \cos \left(\theta_{i}-\theta_{\mathrm{c}}\right)>\cos \frac{\theta_{\mathrm{ca}}}{2} \\ 0, & \text { else }\end{cases}
$$

with $\theta_{\text {ca }}=2 \arcsin \left(\frac{L_{0}}{2 R}\right)$. Since the outer ring speed is 0 , the relative speed of the roller with respect to the outer ring $w_{\text {outer }}(t)$ can be expressed as:

$$
w(t)=w_{\text {outer }}(t)=\frac{1}{2}\left(1-\frac{d}{D} \cos \alpha\right) w_{0}(t)
$$

where $d$ is the roller diameter, $D$ is the bearing pitch diameter.

\subsubsection{Model of inner ring fault}

Fig. 5 shows the case when the inner ring of the bearing has a fault. Different from outer ring fault, the location of the inner ring fault continuously changes as the bearing rotates. Because bearing cage and the inner ring have a relative movement, the inner ring is used as the reference system, which makes it equivalent to the form of the outer ring fault. When a roller rolls over the fault area on the inner raceway, the roller descending depth $c_{\text {inner }}$ between the roller and the raceway can be expressed by: 


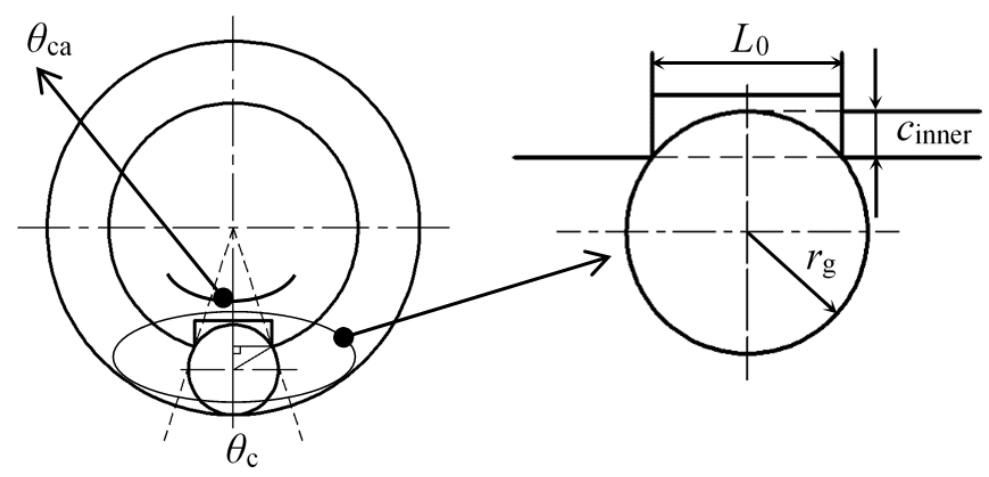

Fig. 5: Model of inner ring fault

$$
c_{\text {inner }}= \begin{cases}r_{\mathrm{g}}-\sqrt{r_{\mathrm{g}}^{2}-\left(\frac{L_{0}}{2}\right)^{2}}, & \cos \left(\theta_{i}-\theta_{\mathrm{c}}\right)>\cos \frac{\theta_{\mathrm{ca}}}{2} \\ 0, & \text { else }\end{cases}
$$

230 with $\theta_{\text {ca }}=2 \arcsin \left(\frac{L_{0}}{2 r}\right)$. Then, the relative speed of the roller with respect 231 to the inner ring $w_{\text {inner }}(t)$ can be calculated as:

$$
w(t)=w_{\text {inner }}(t)=\frac{1}{2}\left(1+\frac{d}{D} \cos \alpha\right) w_{0}(t)
$$

\subsubsection{Model of rolling element fault}
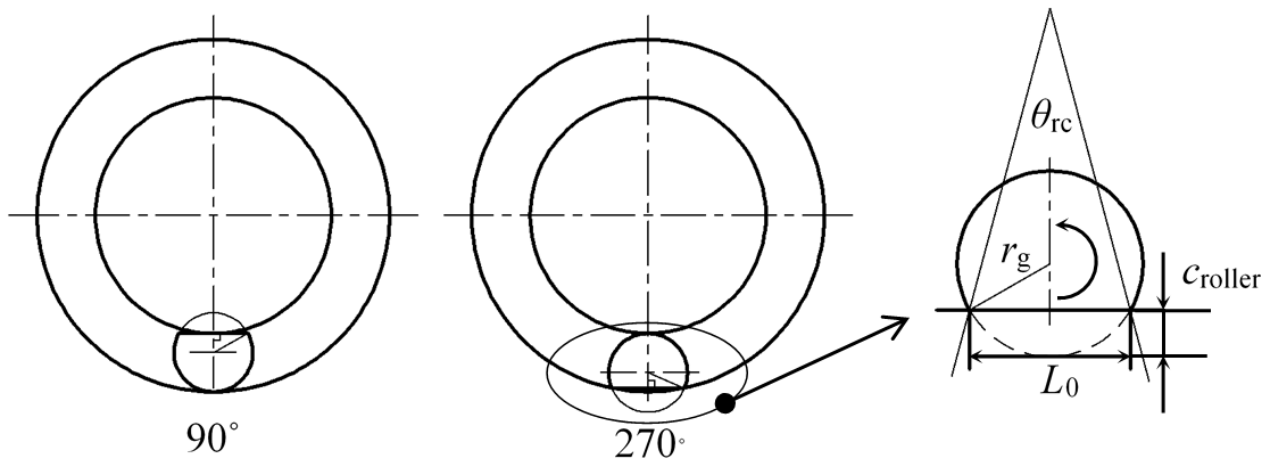

Fig. 6: Model of rolling element fault 
Fig. 6 shows the case when a rolling element of the bearing has a fault, in which $\theta_{\mathrm{rc}}$ is the central angle corresponding to the arc where the fault is located. When the bearing has a rolling element fault, the roller contacts the inner ring raceway and the outer ring raceway once respectively in its own rotation cycle as the inner ring of the bearing rotates. Here, we take the bearing cage as the reference system and assume that the initial angle of the fault area is 0 and the rollers only have rotation (without revolution). When the roller rotates $90^{\circ}$ counterclockwise, it contacts the inner ring. When the roller rotates $270^{\circ}$ counterclockwise, it contacts outer ring. In the process, when the roller rolls over the fault area, the roller descending depth $c_{\text {roller }}$ can be calculated by:

$$
c_{\text {roller }}= \begin{cases}r_{\mathrm{g}}-\sqrt{r_{\mathrm{g}}^{2}-\left(\frac{L_{0}}{2}\right)^{2}}, & \cos \left(\theta_{i}-\phi\right)>\cos \theta_{\mathrm{rc}} \\ 0, & \text { else }\end{cases}
$$

where $\phi=\pi / 2$ and $3 \pi / 2$ for contacting inner and outer raceways, respectively, $\theta_{\mathrm{rc}}=2 \arcsin \left(\frac{L_{0}}{2 r_{0}}\right)$, and the rotating speed of the roller $w_{\text {roller }}(t)$ is given by:

$$
w(t)=w_{\text {roller }}(t)=\frac{D}{2 d}\left(1-\left(\frac{d}{D}\right)^{2}\right) w_{0}(t)
$$

With above analysis, the contact deformation in the fault area under different fault modes can be summarized as:

$$
\delta_{i}=\left(x_{\mathrm{r} 1}-x_{\mathrm{r} 2}\right) \cos \theta_{i}+\left(y_{\mathrm{r} 1}-y_{\mathrm{r} 2}\right) \sin \theta_{i}-c_{0}-c_{\text {change }}
$$

where $c_{\text {change }}$ should be $c_{\text {outer }}, c_{\text {inner }}$ or $c_{\text {roller }}$, depending on the fault mode.

\section{Simulation analysis of the bearing model}

\subsection{Parameters of system}

To verify the proposed model under varying speed, a series simulations are conducted. Tables 1, 2 and 3 list the main parameters of the highspeed EMU axle box bearing, the rotor system, and the unit resonator in the simulation. 
Table 1: The parameter of the bearing

\begin{tabular}{cc}
\hline \hline Parameter & Value of parameter \\
\hline Inner ring mass $m_{1}(\mathrm{~kg})$ & 4.63 \\
Inner ring radius $r(\mathrm{~mm})$ & 6.5 \\
Total bearing mass $m_{2}(\mathrm{~kg})$ & 30 \\
Outer ring radius $R(\mathrm{~mm})$ & 120 \\
Number of roller $N_{0}$ & 17 \\
Contact angle $\alpha\left(^{\circ}\right)$ & 10 \\
Half-cone angle $\beta\left(^{\circ}\right)$ & 1.7 \\
Bearing rolling element diameter $d(\mathrm{~mm})$ & 26.5 \\
Bearing pitch meter $D(\mathrm{~mm})$ & 156.25 \\
Axle load $F(\mathrm{~N})$ & 20250 \\
\hline \hline
\end{tabular}

\subsection{Introduction of GATD}

In order to verify the model, simulation results of the model need to be compared with theoretical results. First, the model is simulated under variable speed conditions to obtain the time-domain waveform of the bearing vibration acceleration. Then, the order ratio spectrum of the vibration is calculated for bearing diagnosis. The model is verified if the order ratio spectrum of the vibration from simulation is close to the theoretical values. Therefore, the key is to find a suitable fault diagnosis method to obtain the order ratio spectrum of bearing vibration acceleration. In 2016, Urbanek et al. [25] proposed a generalized angular temporal deterministic (GATD) signal model for rotating machinery failures under variable speed conditions, based on cyclostationary theory. This GATD signal extracted from wind turbine bearings enables the accurate identification of bearing faults. The advantage of the GATD-based method is that it does not rely on the angle resampling technology and completes the bearing fault diagnosis while retaining the original characteristics of the signal. Therefore, this paper adopts this method to extract the fault characteristics of the model simulation results.

The GATD-based fault diagnosis process is as follows: 
Table 2: The parameter of the bearing system model

\begin{tabular}{cc}
\hline \hline Parameter & Value of parameter \\
\hline Equivalent mass at shaft center $m_{\mathrm{c}}(\mathrm{kg})$ & 274 \\
Bending damping of shaft $C(\mathrm{~N} \cdot \mathrm{s} / \mathrm{m})$ & $2 \times 10^{6}$ \\
Bending stiffness of shaft $\mathrm{K}(\mathrm{N} / \mathrm{m})$ & $1.48 \times 10^{7}$ \\
Mass eccentricity of shaft section $e(\mathrm{~mm})$ & $10^{-5}$ \\
Inner ring damping coefficient $C_{1}(\mathrm{~N} \cdot \mathrm{s} / \mathrm{m})$ & 7000 \\
Inner ring stiffness coefficient $K_{1}(\mathrm{~N} / \mathrm{m})$ & $3.05 \times 10^{8}$ \\
Outer ring damping coefficient $C_{2}(\mathrm{~N} \cdot \mathrm{s} / \mathrm{m})$ & 7000 \\
Outer ring stiffness coefficient $K_{2}(\mathrm{~N} / \mathrm{m})$ & $1.45 \times 10^{10}$ \\
Contact stiffness coefficient $K_{\mathrm{t}}(\mathrm{N} / \mathrm{m})$ & $1.55 \times 10^{10}$ \\
\hline \hline
\end{tabular}

Table 3: The parameter of the unit resonator

\begin{tabular}{cc}
\hline \hline Parameter & Value of parameter \\
\hline Vibrator mass $m_{\mathrm{b}}(\mathrm{kg})$ & 1 \\
Stiffness coefficient $\mathrm{K}_{\mathrm{b}}(\mathrm{N} / \mathrm{m})$ & $8.8826 \times 10^{9}$ \\
Damping coefficient $C_{\mathrm{b}}(\mathrm{N} \cdot \mathrm{s} / \mathrm{m})$ & 9424.8 \\
\hline \hline
\end{tabular}

1. First, the collected faulty bearing signal $x(t)$ is normalized according to the Z-score standardization method:

$$
z_{T}\left(\varphi, f ; \varphi_{0}\right)=\frac{x\left(t-\tau\left(\varphi+\varphi_{0}\right)\right)-\mu_{T}(\varphi)}{\sigma_{T}(\varphi)} w_{T}(t)
$$

where $\varphi(t)$ is the angle that the roller turned at time $t$ with the initial phase $\varphi_{0}, \frac{1}{T}=\max \left(\frac{d \varphi}{d t}\right), w_{T}(t)$ is a Hanning window function, $\tau(\varphi)$ is the angle-fixed time increment used for positioning the window. Here, $\mu_{T}(\varphi)$ is the local angular-temporal mean value of the signal $x(t)$, and $\sigma_{T}(\varphi)$ is the localized angular-temporal standard deviation of $x(t)$, 
which are calculated as:

$$
\begin{gathered}
\mu_{T}\left(\varphi ; \varphi_{0}\right)=\frac{1}{T} \int_{\lambda+T / 2}^{\lambda-T / 2} x(u) \mathrm{du} \\
\sigma_{T}\left(\varphi ; \varphi_{0}\right)=\sqrt{\frac{1}{T} \int_{\lambda+T / 2}^{\lambda-T / 2}\left(x(u)-\mu_{T}(\tau(\varphi))\right)^{2} \mathrm{~d} u}
\end{gathered}
$$

\subsection{Model simulation}

According to theoretical mechanics, the fault characteristic order ratio of the outer ring, the inner ring and the rolling element can be respectively calculated by [26]:

$$
\begin{gathered}
f_{o}=\frac{N_{0}}{2} \times\left(1-\frac{d}{D} \cos \alpha\right) \\
f_{i}=\frac{N_{0}}{2} \times\left(1+\frac{d}{D} \cos \alpha\right) \\
f_{r}=\frac{1}{2} \times \frac{D}{d}\left(1+\left(\frac{d}{D} \cos \alpha\right)^{2}\right)
\end{gathered}
$$


For the bearing used in this research, we have $f_{o}=7.082, f_{i}=9.917$, and $f_{r}=2.871$.

The simulation is conducted in Matlab with a sampling frequency of $512,000 \mathrm{~Hz}$. The angular rotating velocity of the main shaft $w_{0}(t)$ is given in Eq. (26) and shown in Fig. 7. This speed is designed to simulate the entire operating process of the bearing system that contains accelerating process, decelerating process and oscillating process with a sine law. Three simulations are conducted with three types of faults, an outer ring fault, an inner ring fault, and a rolling element fault, all with a fault size $L_{0}=1 \mathrm{~mm}$.

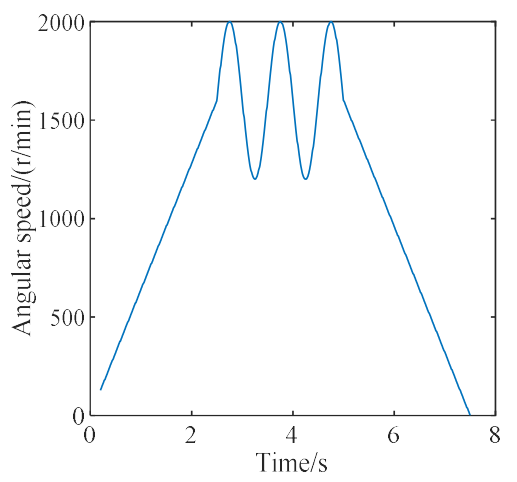

Fig. 7: Angular speed of bearings inner ring

$$
w_{0}(t)= \begin{cases}640 t & 0.2<t \leq 2.5 \\ 1600+400 \sin (\pi(t-2.5)) & 2.5<t \leq 5 \\ 4800-640 t & 5<t \leq 7.5\end{cases}
$$

From the solution of Eqs. (1)-(5), the order ratio spectrum analysis method in [25] is used to obtain the corresponding order ratio spectrum. The time-domain waveform of the acceleration of bearings and the corresponding order ratio spectrum are respectively obtained by simulating the model when an outer ring, an inner ring, and a rolling element fault with $L_{0}=1 \mathrm{~mm}$, respectively. Fig. 8-Fig. 10 show the results.

It can be seen intuitively from the three time domain graph that when a fault occurs, the acceleration amplitude of the bearing changes with the trend of shaft angular speed. In addition, the maximum amplitude increases in order according to the types of the faults, which means the location of the fault has different effects on the vibration of bearing. Apparently, when a fault is on the rolling elements, the bearing has the severest vibration. 


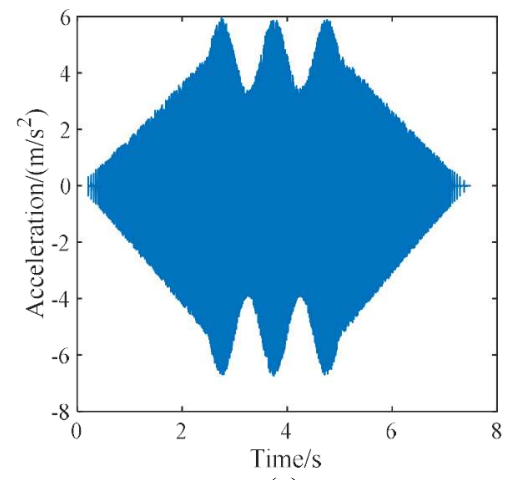

(a)

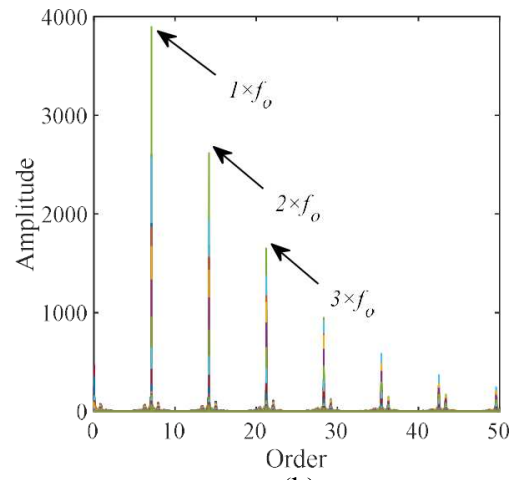

(b)

Fig. 8: Vibration with outer ring fault (a) Vibration signal and (b) order ratio spectrum

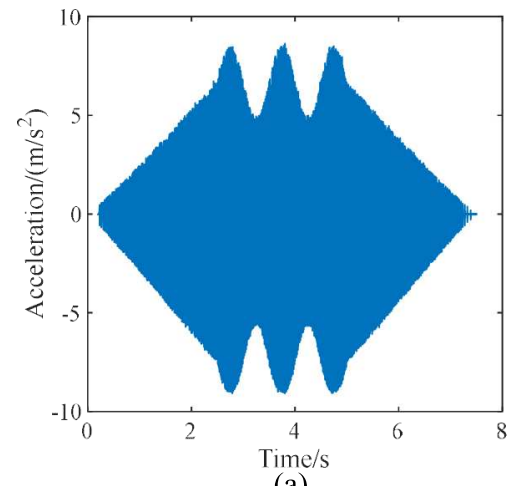

(a)

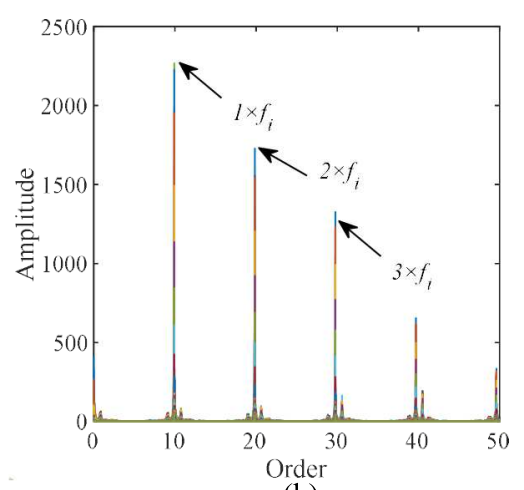

(b)

Fig. 9: Vibration with inner ring fault (a) Vibration signal and (b) order ratio spectrum

Moreover, the results show that the simulated characteristic order ratios of the outer ring (7.805), inner ring (9.9172), and rolling element (2.8694) are close to the calculation results given by Eq. (23)-(25). At the same time, the corresponding frequency multiplication can be observed in the order ratio spectrum, which proves the correctness of the model.

Since the order ratio spectrum is obtained by the signal after two Fourier transforms, there is a linear relationship between the order ratio spectrum amplitude and the original signal amplitude. Letting the amplitude corresponding to the single frequency (ACSF) in the signal order ratio spectrum under an outer ring fault and an inner ring fault are $A_{\mathrm{o}}$ and $A_{\mathrm{i}}$, respectively. The influence of the fault location and the fault size on the ACSF is studied and the results are shown in Fig. 11. 


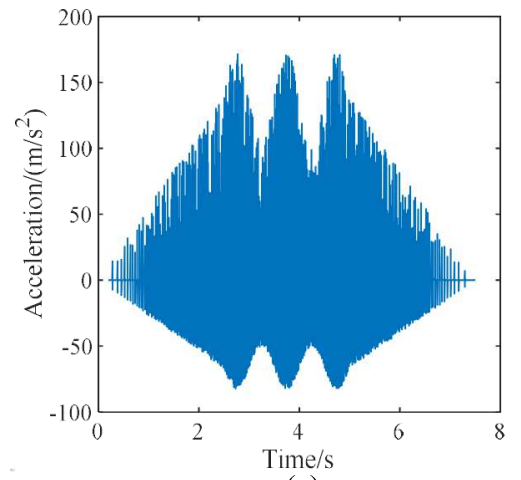

(a)

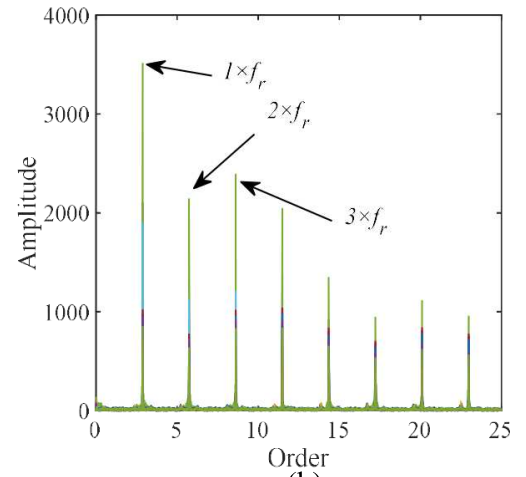

(b)

Fig. 10: Vibration with rolling element fault (a) Vibration signal and (b) order ratio spectrum

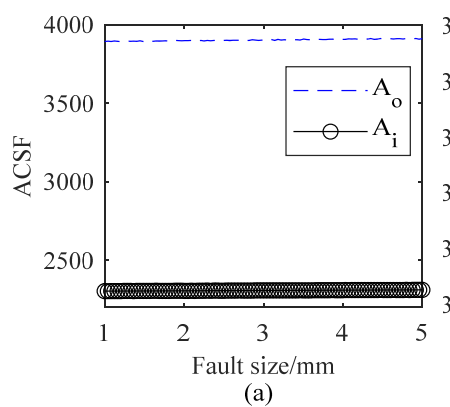

(a)

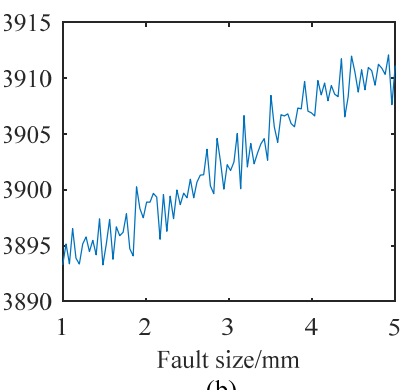

(b)

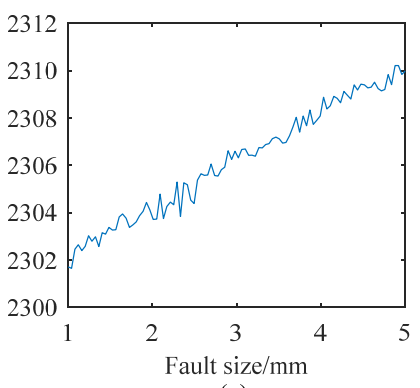

(c)

Fig. 11: Result of amplitude change with fault size

Fig. 11(a) shows the ACSF values under outer ring is always larger than that under inner ring. Fig. 11(b) and (c) show that the ACSF values in the order ratio spectrum under these two fault conditions increase as the fault sizes increases, which indicates that the fault size has an important influence on the motion state of the bearing. As the size of the fault increases, more energy is generated in the fault area when the roller collides with the raceway, which causes the increase of the amplitude at this frequency.

\section{Experimental verification}

To make the operating condition close to real operation, the experiments use a speed profile in which the bearing rotating speed is accelerated from 0 to a certain speed, maintained for a period of time, and then decelerated to 
0 . It is important to note that, in the experiments, tachometer is not used to measure the speed of the bearing and no program is used to control its speed. For the faulty type verification, the vibration is from experiment and speed profile is not needed. However, the speed profile is used as the model input for the bearing operating condition verification. Since the model speed profile is unknown, the instantaneous speed of the bearing must be estimated from the bearing vibration signals.

\subsection{Fault type verification}

Two EMU axle box bearings are used in the experiments. One bearing has a through-groove fault on the outer ring with the width of $L_{0}=1 \mathrm{~mm}$ and the other one has a pitting fault on the inner ring with $L_{0}=0.1 \mathrm{~mm}$. The schematic diagram of the outer ring, the fault on the outer ring, and the fault on the inner ring are shown in Fig. 12. In the experiments, the vibration signal of the bearing is collected in $60 \mathrm{~s}$ with a sampling frequency of 51,200 $\mathrm{Hz}$.
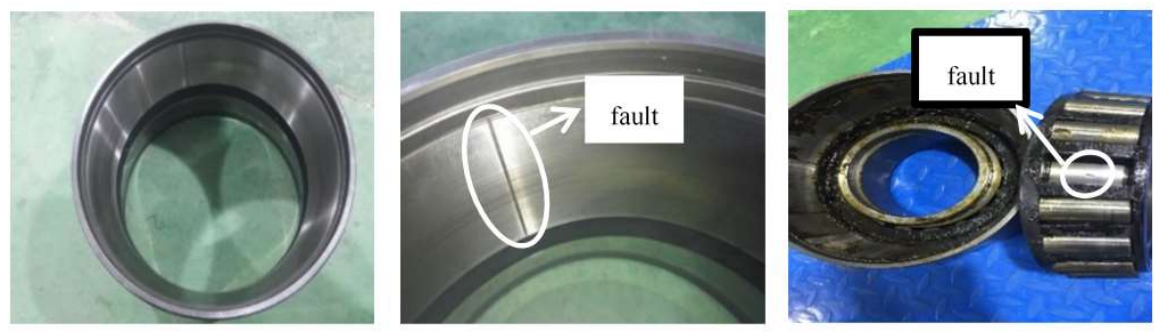

Fig. 12: The outer ring, outer ring fault, and inner ring fault of the bearing

The acceleration sensors should be installed near the experimental bearing so that the vibration signal can be accurately measured. Fig. 13 shows the experimental platform and the horizontal and vertical location of the sensors.

Experiments are carried out on the bearings with seeded faults. For the bearing with the outer ring fault, Fig. 14 shows the time domain signal and the order ratio spectrum of the bearing vibration acceleration. It can be seen that the difference of the experimental results and the calculation results of the first three-order fault feature order ratio are respectively $5.97 \%, 5.67 \%$ and $6.34 \%$.

For the bearing with an inner ring fault, Fig. 15 shows the time domain signal and order ratio spectrum of the bearing vibration acceleration. The 

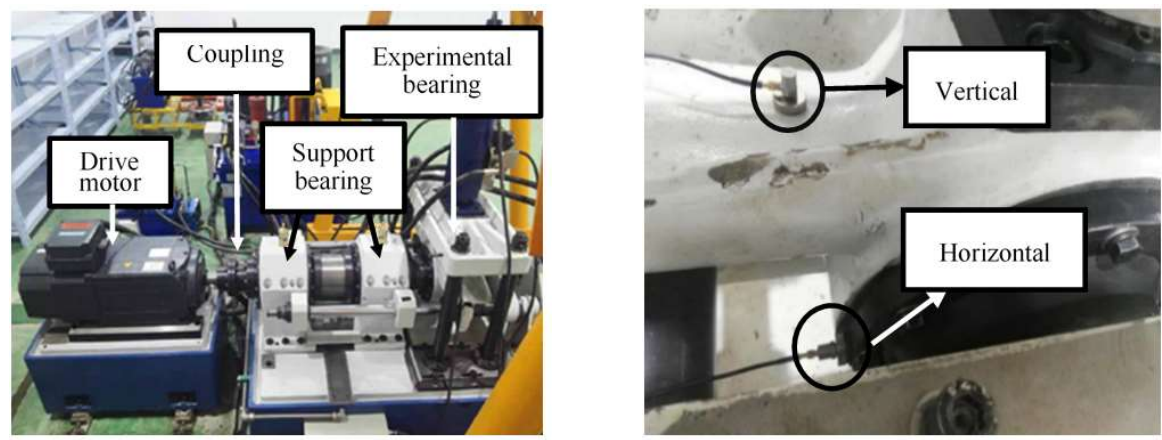

Fig. 13: Test rig of roller bearing of railway vehicle and sensors' location

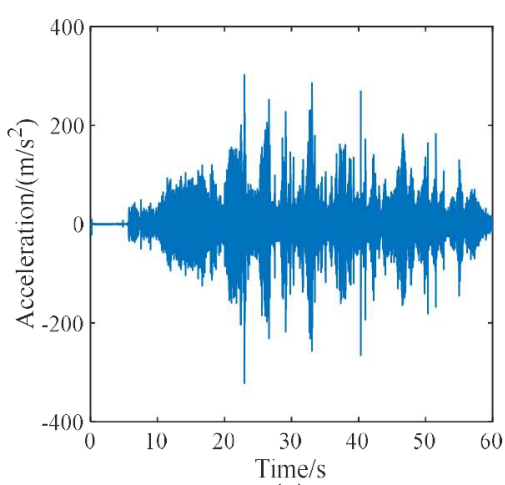

(a)

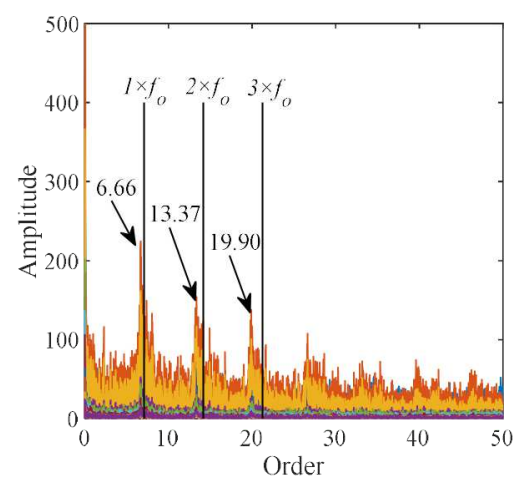

(b)

Fig. 14: Vibration with outer ring (a) Time domain vibration signal, (b) order ratio spectrum

difference of the experimental results and the calculation results of the first three-order fault feature order ratio are respectively $2.59 \%, 3.95 \%$ and $4.15 \%$.

As mentioned early, the bearing speed is changing in the experiments. Under these non-linear operating conditions, the acceleration amplitude of the bearing changes continuously with time, which is a typical variable speed bearing operation for high-speed trains. The results show that, compared with the theoretical value given by Eq. (23)-(24), the difference of the fault characteristic order ratio under two experimental conditions are within the allowable range, which proves the effectiveness of the model.

\subsection{Bearing operating conditions verification}

In order to further prove that the model is also effective in real working conditions and has a certain anti-interference ability against noise, the 

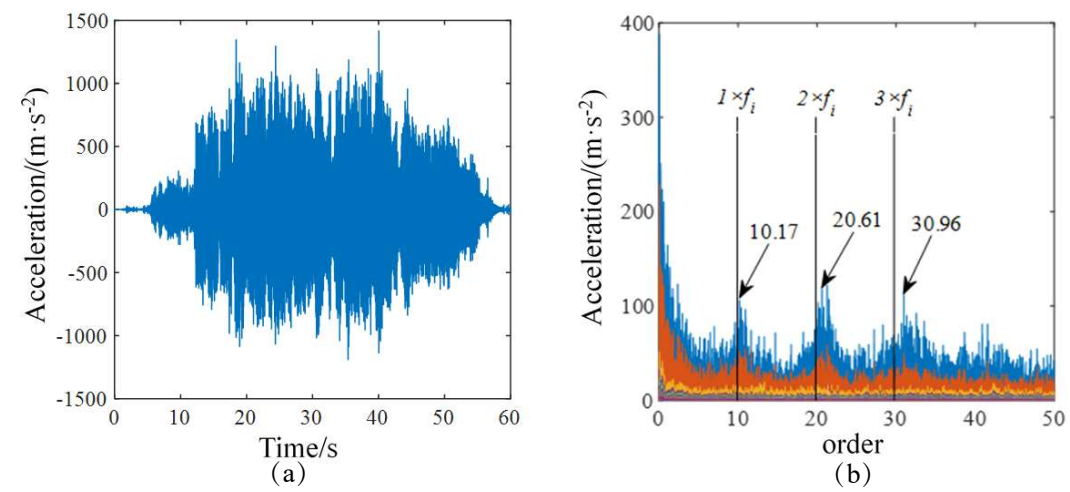

Fig. 15: Vibration with inner ring (a) Time domain vibration signal, (b) order ratio spectrum

bearing speed estimated in the experiment is used as the input of the model and noise is added to the bearing vibration acceleration signal obtained from the model. The order ratio spectrum analysis is performed on the model output vibration signal with noise. If the model result is consistent with the calculation result, the model is verified.

Since there is no speed measuring device, such as a tachometer, and speed profile is unknown, the real-time speed of the bearing spindle cannot be obtained. Therefore, this study extracts the instantaneous speed curve from the signals collected in the two experiments, and uses a time function to fit this two working conditions for model speed input. At the same time, on-site noise is added to the model to make the simulation close to a real noisy environment.

\subsubsection{Verification under outer ring fault}

The speed curve is extracted from the experimental signals by the method in [27], and the noise signal is extracted from the signal using the wavelet noise reduction method [28]. Fig. 16 shows the instantaneous speed and experimental site noise extracted from the outer ring fault test signal. The fitted curve function is $f(t)=-0.002 t^{4}+0.2535 t^{3}-11.7572 t^{2}+232.4658 t-$ $2.0618 \times 10^{-4}$.

It can be seen that the bearing speed experiences a process of speed increase, slowly change, and decrease during the experiment. Meanwhile, the noise amplitude experience the same process as well.

With the proposed model, the fitted speed curve, and the noise, Fig. 


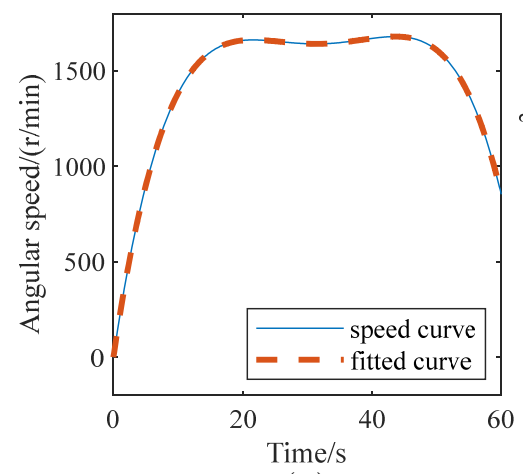

(a)

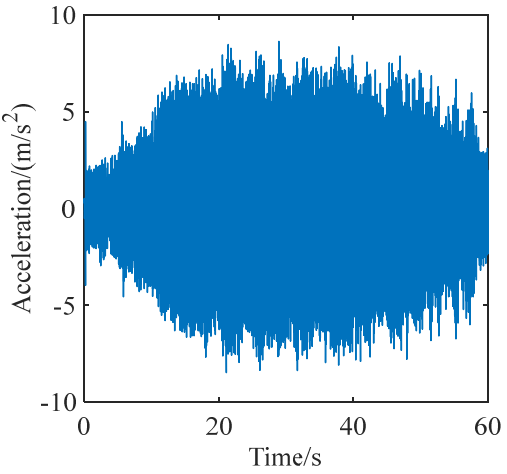

(b)

Fig. 16: Experimental signal under outer ring fault (a) speed curve, (b) site noise

17(a) shows the vibration signal obtained from the model. The order ratio spectrum in Fig. 17(b) shows that the values of frequency-singled, frequencydoubled and frequency-tripled are close to the theoretical values given by Eq. (23). The results demonstrate the effectiveness of the model.

\subsubsection{Verification under inner ring fault}

The same methods are used to obtain the time frequency curve and experimental site noise in inner ring fault experiment. Fig. 18 shows the instantaneous speed and experimental site noise extracted from the inner ring fault test signal. The fitted curve function is $f(t)=-0.0028 t^{4}+0.3423 t^{3}-$ $14.3913 t^{2}+251.987 t-6.0213 \times 10^{-4}$.

With the proposed model, the vibration signal of the model shown in Fig. 19(a) under the fitted speed profile given in Fig. 18(a) can be obtained. The order ratio spectrum in Fig. 19(b) shows that the values of frequency-singled, frequency-doubled and frequency-tripled are close to the theoretical values given by Eq. (24). The results demonstrate the effectiveness of the model.

It is noticed that the ACSF values under outer ring fault is smaller. The reason is that, when the bearing speed changes, the instantaneous impact load and amplitude caused by a single fault are reduced. Meanwhile, There are very few messy frequency components in the order ratio spectrum, which proves that the model has good anti-interference ability. Even if the experimental site noise is added, the simulation model can get very obvious results. 


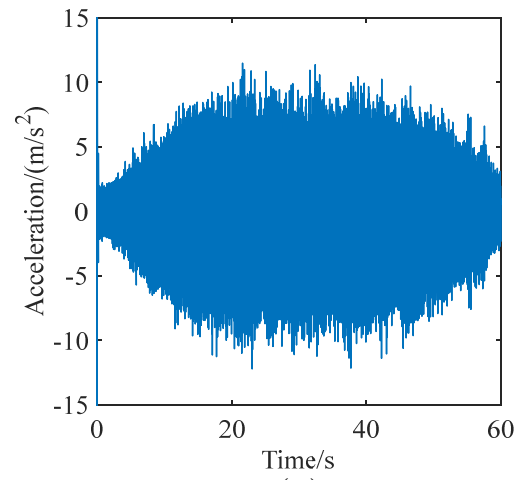

(a)

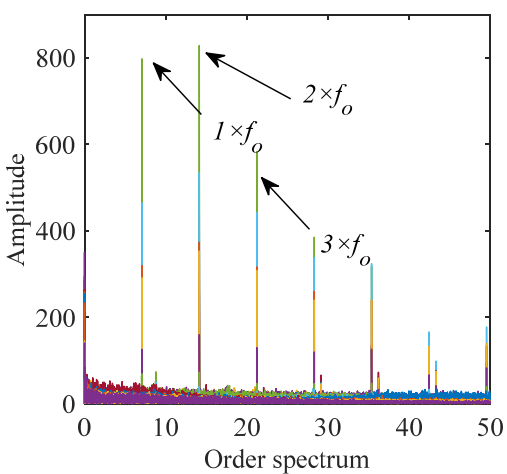

(b)

Fig. 17: Simulation results of bearing with outer ring fault (a) vibration acceleration, (b) order ratio spectrum

\section{Conclusion}

This paper establishes a dynamic model of bearing systems in the running part of high-speed trains under variable speed conditions. The novelty is that an angle iteration method is used to address the problem that the roller space position cannot be determined in bearing rotation, which is ignored in the existing researches. By simulating the actual operating conditions of the train, the vibration response of the system in the process of speed increase, decrease and speed oscillation is obtained. The results show that the vibration acceleration amplitude of bearing outer ring is positively correlated with bearings spindle speed. To demonstrate the effectiveness of the model, bearing experiments and simulations under outer ring fault and inner ring fault with varying speed are conducted. The order ratio of the fault characteristics show that the errors between the simulation and experimental results are always within the allowable range, which demonstrate the effectiveness of the model.

\section{Acknowledgement}

The authors would like to thank X Gu, W Liu and L Zhao for their work on the fault bearing experiment of high-speed train. Many thanks to the anonymous reviewers, who provided valuable feedback and recommendations. 


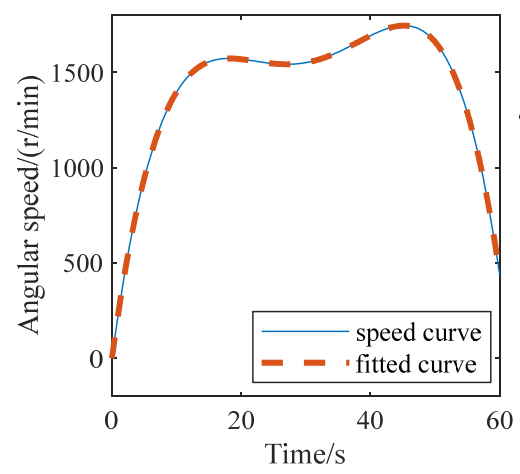

(a)

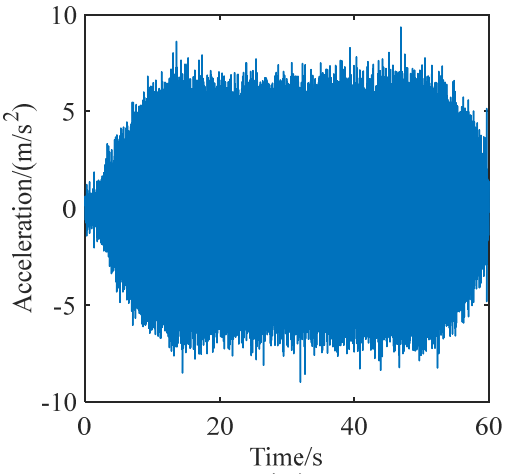

(b)

Fig. 18: Experimental signal under inner ring fault (a) speed curve, (b) site noise

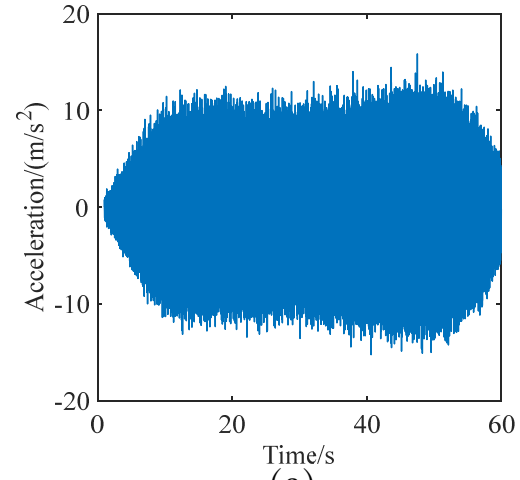

(a)

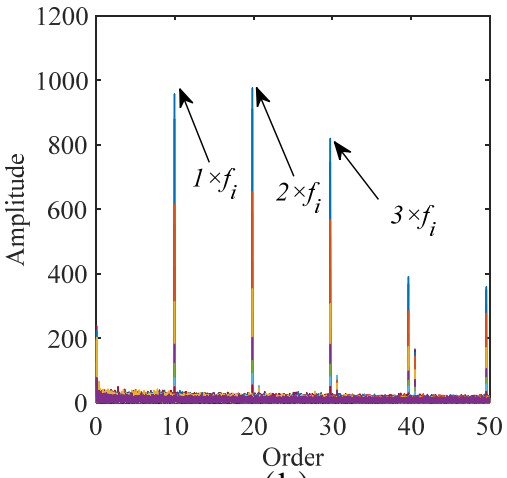

(b)

Fig. 19: Simulation results of bearing with inner ring fault (a) vibration acceleration, (b) order ratio spectrum

\section{Authors' contributions}

Yongqiang Liu: Validation, Resources, Writting- Reviewing and Editing.

Baosen Wang: Conceptualization, Methodology, Coding, Writting- Original draft and Editing.

Bin Zhang: Conceptualization, Resources, Investigation, Writting- Reviewing and Editing.

Shaopu Yang: Validation, Writting- Reviewing and Editing. 


\section{Authors' information}

Yongqiang Liu, born in 1983, is currently a professor at School of Mechanical Engineering, Shijiazhuang Tiedao University, China. He received his phD degree in vehicle engineering from Beijing Jiaotong Unviversity, China, in 2011. His research interests include prognostics and health management, vehicle system dynamics, and smart soft material and damper.

Baosen Wang, born in 1990, is currently a phD candidate at School of Traffic and Transportation, Shijiazhuang Tiedao University, China. He received his master's degree from Shijiazhuang Tiedao University, China, in 2018. His research interests include dynamic modeling analysis and bearing fault diagnosis of high-speed train bearings.

Bin Zhang, born in 1972, is currently a associate professor at Department of Electrical Engineering, University of South Carolina, USA. He received his phD degree in electrical engineering from Nanyang Technological University, Singapore, in 2007. His research interests include prognostics and health management, intelligent systems and controls, and their applications to various engineering systems.

Shaopu Yang, born in 1962, is currently a professor at State Key Laboratory of Mechanical Behavior and System Safety of Traffic Engineering Structures, Shijiazhuang Tiedao University, China. He received his phD degree in mechanics from Tianjin University, China, in 1991. His research interests include dynamics and control of vehicle system, theory and application of nonlinear system dynamics and control.

\section{Funding}

Supported by the National Natural Science Foundation of China (Nos. 11790282,12032017,11802184,11902205 and 12002221), S\&T Program of Hebei (20310803D), and Natural Science Foundation of Hebei Province (No A2020210028), Postgraduates Innovation Foundation of Hebei Province (CXZZBS2019154), and State Foundation for Studying Abroad.

\section{Competing interests}

The authors declare no competing financial interests. 


\section{Author Details}

1. School of Mechanical Engineering, Shijiazhuang Tiedao University, Shijiazhuang, 050043, China.

2. School of Traffic and Transportation, Shijiazhuang Tiedao University, Shijiazhuang, 050043, China.

3. College of engineering and computing, University of South Carolina, Columbia, South Carolina, 29208, America.

4. State Key Laboratory of Mechanical Behavior and System Safety of Traffic Engineering Structures, Shijiazhuang Tiedao University, Shijiazhuang 050043, China.

\section{References}

[1] J.-W. Yang, J.-H. Wang, Q. Huang, M. Zhou, Reliability assessment for the solenoid valve of a high-speed train braking system under small sample size, Chinese Journal of Mechanical Engineering 31 (2018) 1-11.

[2] Y. Yu, J. Liu, B. Ye, G. Yu, X. Jin, L. Sun, J. Tong, Design and experimental research on seedling pick-up mechanism of planetary gear train with combined non-circular gear transmission, Chinese Journal of Mechanical Engineering 32 (2019) 1-13.

[3] Z. Shang, W. Li, M. Gao, X. Liu, Y. Yu, An intelligent fault diagnosis method of multi-scale deep feature fusion based on information entropy, Chinese Journal of Mechanical Engineering 34 (2021) 1-16.

[4] M. Chinta, A. Palazzolo, Stability and bifurcation of rotor motion in a magnetic bearing, Journal of sound and vibration 214 (1998) 793-803.

[5] J.-J. Sinou, Non-linear dynamics and contacts of an unbalanced flexible rotor supported on ball bearings, Mechanism and Machine Theory 44 (2009) 1713-1732.

[6] S. Fukata, E. H. Gad, T. Kondou, T. Ayabe, H. Tamura, On the radial vibration of ball bearings: computer simulation, Bulletin of JSME 28 (1985) 899-904.

[7] M. Tiwari, K. Gupta, O. Prakash, Effect of radial internal clearance of a ball bearing on the dynamics of a balanced horizontal rotor, Journal of sound and vibration 238 (2000) 723-756. 
[8] N. Sawalhi, R. Randall, Simulating gear and bearing interactions in the presence of faults: Part i. the combined gear bearing dynamic model and the simulation of localised bearing faults, Mechanical Systems and Signal Processing 22 (2008) 1924-1951.

[9] N. Sawalhi, R. Randall, Simulating gear and bearing interactions in the presence of faults: Part ii: Simulation of the vibrations produced by extended bearing faults, Mechanical Systems and Signal Processing 22 (2008) 1952-1966.

[10] N. A. H. Tsuha, K. L. Cavalca, Stiffness and damping of elastohydrodynamic line contact applied to cylindrical roller bearing dynamic model, Journal of Sound and Vibration (2020) 115444.

[11] S. Xi, H. Cao, X. Chen, Dynamic modeling of spindle bearing system and vibration response investigation, Mechanical Systems and Signal Processing 114 (2019) 486-511.

[12] Q. Cao, X. Guo, G. Xiong, Research on dynamic characteristics of loose support system of rolling bearing of high-speed train, Mechanical Engineering 52 (2016) 87-95.

[13] H. Cao, Z. He, Y. Zi, Mechanical characteristic modeling and damage mechanism analysis of high-speed rolling bearings, Chinese Vibration Engineering Society 31 (2012) 134-140.

[14] t. Yang, Q. Ding, T. Guo, Vibration characteristics analysis of local fault on emu train bogie bearing, Vibration Measurement and Diagnosis 36 (2016) 665-673.

[15] Y. Liu, B. Wang, S. Yang, Nonlinear dynamic behaviors analysis of bearings rotor system with outer ring faults in the high-speed train, Mechanical Engineering 54 (2018) 17-25.

[16] Z. Wang, Y. Song, Z. Yin, R. Wang, W. Zhang, Random response analysis of axle-box bearing of a high-speed train excited by crosswinds and track irregularities, IEEE Transactions on Vehicular Technology 68 (2019) 10607-10617. 
[17] C. Mishra, G. Chakraborty, A. K. Samantaray, Rolling element bearing fault modelling to develop a diagnosis scheme for oscillating and nonuniform shaft rotation, Proc. INACOMM 2013 (2013) 86-94.

[18] C. Mishra, A. Samantaray, G. Chakraborty, Bond graph modeling and experimental verification of a novel scheme for fault diagnosis of rolling element bearings in special operating conditions, Journal of Sound and Vibration 377 (2016) 302-330.

[19] L. Ma, J.-h. Zhang, J.-w. Lin, J. Wang, X. Lu, Dynamic characteristics analysis of a misaligned rotor-bearing system with squeeze film dampers, Journal of Zhejiang University-SCIENCE A 17 (2016) 614-631.

[20] L. Cui, Y. Zhang, F. Zhang, J. Zhang, S. Lee, Vibration response mechanism of faulty outer race rolling element bearings for quantitative analysis, Journal of Sound and Vibration 364 (2016) 67-76.

[21] Y. Qin, F. Cao, Y. Wang, W. Chen, H. Chen, Dynamics modelling for deep groove ball bearings with local faults based on coupled and segmented displacement excitation, Journal of Sound and Vibration 447 (2019) 1-19.

[22] D. Xiang, Y. Shen, Y. Wei, A contact force model considering meshing and collision states for dynamic analysis in helical gear system, Chinese Journal of Mechanical Engineering 32 (2019) 1-12.

[23] Y. Jiang, W. Huang, J. Luo, W. Wang, An improved dynamic model of defective bearings considering the three-dimensional geometric relationship between the rolling element and defect area, Mechanical Systems and Signal Processing 129 (2019) 694-716.

[24] A. M. Ahmadi, D. Petersen, C. Howard, A nonlinear dynamic vibration model of defective bearings-the importance of modelling the finite size of rolling elements, Mechanical Systems and Signal Processing 52 (2015) 309-326.

[25] J. Urbanek, T. Barszcz, A. Jablonski, Application of angular-temporal spectrum to exploratory analysis of generalized angular-temporal deterministic signals, Applied Acoustics 109 (2016) 27-36. 
[26] Y. Hu, X. Tu, F. Li, H. Li, G. Meng, An adaptive and tacholess order analysis method based on enhanced empirical wavelet transform for fault detection of bearings with varying speeds, Journal of Sound and Vibration 409 (2017) 241-255.

[27] Y. Yang, Z. Peng, G. Meng, W. Zhang, Spline-kernelled chirplet transform for the analysis of signals with time-varying frequency and its application, IEEE Transactions on Industrial Electronics 59 (2011) 16121621.

[28] S. Wen, W.-S. Gan, D. Shi, An improved selective active noise control algorithm based on empirical wavelet transform, in: ICASSP 20202020 IEEE International Conference on Acoustics, Speech and Signal Processing (ICASSP), IEEE, 2020, pp. 1633-1637. 